\title{
Spongiotic reaction patterns in autoimmune bullous dermatoses (Review)
}

\author{
RALUCA ANCA BALAN, LUDMILA LOZNEANU, ADRIANA GRIGORAS, IRINA DRAGA CARUNTU, \\ TEODORA ANA BALAN, SIMONA ELIZA GIUSCA and CORNELIA AMALINEI
}

Department of Morphofunctional Sciences I, ‘Grigore T. Popa’ University of Medicine and Pharmacy, 700115 Iasi, Romania

Received July 7, 2021; Accepted August 6, 2021

DOI: $10.3892 / \mathrm{etm} .2021 .10769$

\begin{abstract}
Spongiosis or a spongiotic reaction pattern is the histological hallmark of intercellular epidermal edema, viewed as clear spaces within the epidermis. Although considered a histopathological term, spongiosis has clinical correlations, with the variable degrees of spongiotic reaction leading to different dermatological findings. This review aimed to highlight the spongiotic reactive patterns found in different autoimmune bullous dermatoses, considering the paucity of publications in this domain. The pathogenesis of spongiosis assumes the passage of extravasated edema fluid from the dermis into the epidermis, frequently accompanied by dermal inflammatory cells, and classification of the spongiotic reaction patterns, as well as their associated spongiotic dermatitis, take into consideration the type and distribution of these inflammatory cells. It is mandatory to consider different reactive processes, specific for other skin disorders, which act as simulants of different spongiotic patterns for the diagnosis. Considering the possible transient occurrence, the heterogeneity and non-specificity of the histopathological features of these diseases, the diagnosis is very complex, requiring clinicopathological correlations and additional analyses. A deep insight into spongiosis pathogeny may open the perspectives of a classification refinement of autoimmune bullous dermatoses.
\end{abstract}

Correspondence to: Dr Raluca Anca Balan or Professor Cornelia Amalinei, Department of Morphofunctional Sciences I, 'Grigore T. Popa' University of Medicine and Pharmacy, 16 Universitatii Street, 700115 Iasi, Romania

E-mail: raluca.balan@umfiasi.ro

E-mail: cornelia.amalinei@yahoo.com

Key words: spongiotic reaction pattern, intraepidermal autoimmune bullous dermatoses, subepidermal autoimmune bullous dermatoses, eosinophilic spongiosis, neutrophilic spongiosis

\section{Contents}

1. Introduction

2. Pathogenesis

3. Classification of the spongiotic reaction patterns

4. Mimickers of the spongiotic tissue reaction

5. Spongiosis patterns in autoimmune bullous diseases

6. Conclusions

\section{Introduction}

Spongiosis or spongiotic reaction pattern (classical eczematous tissue reaction) represents the histological hallmark of intercellular epidermal edema, translated by cell condensation with corresponding wide spaces between keratinocytes, with elongated intercellular bridges ('spinous processes'), leading to a sponge-like appearance of the epidermis (1-4). Hematoxylin and eosin (H\&E) routine staining highlights this process as clear spaces within the epidermis. Sometimes, when there is a marked intraepidermic edema, spongiosis forms multiloculated vesicles. Moreover, spongiosis includes also intracellular edema.

Although considered a histopathological term, spongiosis has clinical correlations, with the variable degrees of spongiotic reaction leading to different dermatological findings, from mild skin erythema to crusted plaques, oozing papulovesicles or vesiculobullous lesions, with a specific collaret of scale for resolving lesions (1-3). However, the most common cutaneous lesion associated with spongiosis is eczematous dermatitis (5).

Autoimmune bullous dermatoses present limited spongiotic reactive patterns, the various dermatoses practically exhibiting the same type of spongiosis.

This review aimed to provide a summarized overview of the current state of research regarding spongiotic patterns found in autoimmune bullous dermatoses, considering that only few publications have this approach on spongiosis. For this review, a literature search was conducted using the main scientific databases (Web of Science, Scopus, Science Direct, Google Scholar, Cochrane Database for Systematic Reviews, PubMed/Medline) and acquisition was based on a database search using the following keywords: 'spongiosis', 'autoimmune bullous dermatoses', 'spongiotic pattern' in all the relevant combinations. In the next step of selection, 
all the relevant full content English written literature in a time frame of 20 years was categorized, using as inclusion criteria only autoimmune bullous diseases. Consequently, 25 studies and 13 book chapters were selected and used in this review.

\section{Pathogenesis}

Still controversial and with limited studies, the mechanism of spongiosis assumes the passage of extravasated edema fluid from the dermis into the epidermis (1). The dermal inflammatory cells, which usually accompany this process, with/without epidermal involvement, probably secrete substances that regulate the particular tissue fluid dynamic (1). The classification of the spongiotic reaction patterns, as well as their associated spongiotic dermatitis, take into consideration the type and distribution of these inflammatory cells.

Although widely accepted, the fluid extravasation etiology, as well as the mechanism of dermal extravascular fluid influx into the epidermis, remain to be understood (4). Two main opposing concepts have been developed, one related to the osmotic gradient towards the epidermis, and the other considering that epidermal involvement of edema is due to increased dermal hydrostatic pressure (4). Even though opposite, both mechanisms seem to be involved, as spongiotic reaction represents a dynamic process, with transient vesicles formed in different locations in the epidermis (4). Overlying parakeratosis may occur because of disordered maturation or of an accelerated keratinocyte movement towards the superficial layers. When involved by plasma droplets, parakeratosis is responsible for Collarette scales, which appear in resolving lesions (4).

Studies have classically shown that spongiosis is caused by the cleavage of membrane E-cadherins due to interferon (IFN)- $\gamma$-producing $\mathrm{T}$ cell-mediated keratinocyte apoptosis, which affects the integrity of E-cadherins but retains desmosomal cadherins, in acute eczematous dermatitis $(6,7)$. Maintaining intercellular contact only at the desmosomal level confers the sponge-like morphology to the epidermis $(4,6,7)$. Recent data, which have demonstrated high expression levels of interleukin (IL)-4 and IL-13 in biopsy specimens of allergic contact dermatitis, suggest that IFN- $\gamma$ is not the only trigger of spongiosis pathogenesis $(6,8)$.

Moreover, Ohtani et al demonstrated that in acute eczema, the intercellular space of spongiotic epidermis contains a high amount of hyaluronan (HA). By histochemistry, they demonstrated that the intercellular space enlargement related to acantholysis is not associated with an abnormal HA quantity, suggesting that, in pemphigus vulgaris, impairment of keratinocyte cohesion does not directly cause excessively accumulation of HA (6). At the same time, the study observed that not all skin diseases characterized by lymphocyte exocytosis have more HA in the spongiotic epidermis (6).

Although dermatomyositis exhibits interface dermatitis in almost all cases, several patients were identified who showed spongiotic dermatitis on skin biopsies, the histological aspect being more correlated with eczematous lesions (9). In this regard, one case-control study demonstrated that increased immunohistochemical expression of myxovirus-resistance protein A (MxA), IFN- $\beta$, CD11c, and dendritic cell lectin (BDCA2) protein in dermatomyositis-related spongiotic dermatitis can represent a useful differential diagnostic tool between dermatomyositis and eczema (9).

\section{Classification of the spongiotic reaction patterns}

The epidermis intercellular edema comprises six main spongiotic reaction patterns, each correlated with different skin diseases, the major ones being atopic dermatitis, contact dermatitis, nummular and seborrheic dermatitis. These entities present psoriasiform hyperplasia, which accompanies spongiosis, sometimes in the same biopsy, with different stages of activity. The areas of spongiosis may vary from microscopic foci to grossly observable vesicles or bullae (Table I) (3).

The spongiotic reactive patterns include: i) neutrophilic spongiosis, with neutrophils within foci of spongiosis; ii) eosinophilic spongiosis, where the spongiosis foci contain numerous eosinophils; iii) miliarial (acrosyringial) spongiosis, with edema related to the acrosyringium; iv) follicular spongiosis, with edema involving the follicular infundibulum; v) pityriasiform spongiosis, characterized by small vesicles with lymphocytes, histiocytes, and Langerhans cells; and vi) haphazard spongiosis, with no particular pattern. There is a variant of the haphazard type, considered as a seventh pattern of spongiosis, characterized by an association of epidermal spongiosis and subepidermal edema, with different degrees of severity (Table II) (3).

Although displaying several histological forms, spongiosis shows limited patterns in bullous autoimmune diseases, without a characteristic morphological type.

Considering these aspects, the spongiotic reactions represent one of the most difficult histopathological patterns that can lead to a specific clinicopathological diagnosis (3).

\section{Mimickers of the spongiotic tissue reaction}

There are different reactive processes, specific for other skin disorders, which act as simulants of different spongiotic patterns.

Variants of lupus erythematosus, pityriasis lichenoides, erythema multiforme or fixed drug eruption, with vacuolar changes or lichenoid patterns, may exhibit mild suprabasal spongiosis.

Some viral diseases, such as morbilliform drug eruptions or exanthemas show epidermal basal spongiosis, while others, such as herpes zoster and simplex, display ballooned degenerated keratinocytes followed by acantholysis, changes that obscure the mild spongiotic reaction. Early psoriasis, which is not a spongiotic disease, may show some epidermal spongiosis close to the dermal papillae (3). Another mimicker of spongiosis is a high amount of acid mucopolysaccharides accumulated in the follicular infundibulum of follicular mucinosis, identified with mucin stains. The accumulation of mononuclear cells in spongiotic dermatitis can sometimes simulate the Pautrier microabscesses found in mycosis fungoides. The difference resides, on the one hand, in the specific vase-like shape of intraepidermal inflammatory cell collections, with their extremities situated between granular and keratinized layers, and on the other hand, in the characteristic phenotypes expressed by mononuclear cells: CD1a, CD36, CD38 and S100 protein (3). 


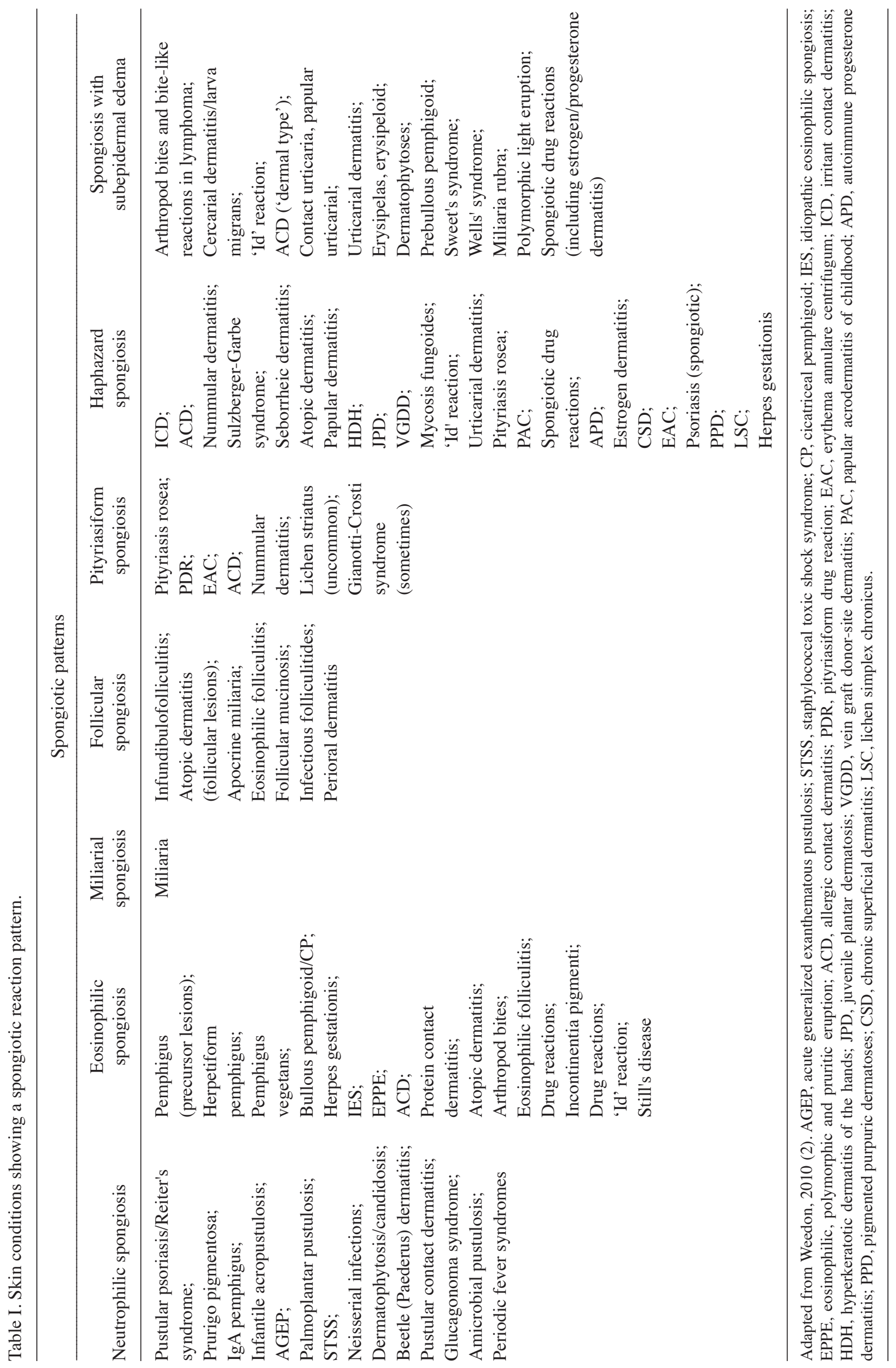


Table II. Types of spongiotic reaction patterns and their histological characteristics.

Spongiotic pattern

Histological characteristics

\author{
Neutrophilic spongiosis \\ Eosinophilic spongiosis \\ Miliarial (acrosyringial) spongiosis \\ Follicular spongiosis \\ Pityriasiform spongiosis \\ Haphazard spongiosis \\ Variant of haphazard spongiosis
}

\begin{abstract}
Neutrophils in areas of spongiosis
Eosinophils within areas of spongiosis

Edema correlated with the acrosyringium

Spongiotic foci involving the follicular infundibulum

Small vesicles with lymphocytes, histiocytes, and Langerhans cells

No particular pattern

Epidermal spongiosis with subepidermal edema, mild to severe variants, can evolve towards subepidermal blisters
\end{abstract}

\section{Spongiosis patterns in autoimmune bullous dermatoses}

Autoimmune bullous dermatoses represent a group of diseases, which develop autoantibodies against structural adhesion proteins from the epidermis and basement membrane area, with the formation of skin or mucosal blisters $(10,11)$. The diagnosis is very complex due to their heterogeneity, always associating different diagnostic methods such as direct or indirect immunofluorescence, immunoblot analysis, immunohistochemistry, or molecular biology to histopathological and clinical assessment $(10,11)$.

The classification of autoimmune bullous dermatoses takes into consideration the histologic localization of the bullae, dividing this category in intraepidermal and subepidermal bullous dermatoses.

Intraepidermal autoimmune bullous dermatoses. Intraepidermal autoimmune bullous dermatoses include five subtypes of the pemphigus category: pemphigus vulgaris (PV), pemphigus foliaceus (PF), paraneoplastic pemphigus (PNP), IgA pemphigus, and pemphigus herpetiformis (PH) (11).

Histopathologically, intraepidermal bullae or pustules characterize these dermatoses, aspects observed also in various diseases with similar histology but different etiopathogeny (11). The intercellular or intracellular edema (spongiosis or ballooning degeneration) can cause desmosomes destruction (11).

Pemphigus vulgaris $(P V)$. $\mathrm{PV}$ is a chronic autoimmune intraepithelial bullous dermatosis, characterized by $\mathrm{IgG}$ autoantibodies directed against transmembrane cadherin desmoglein3 (Dsg3) $(10,12,13)$. Classically, the diagnosis considered lesional histopathological assessment and perilesional direct immunofluorescence, but recently, ELISA tests have been identified as the most accurate technique for the diagnosis of PV $(10,13)$.

The key histopathological feature of pemphigus group diseases is the intraepidermal bullae with acantholysis, which makes clinical correlations and immunofluorescence techniques mandatory for differential diagnosis with other intraepidermal bullous diseases $(11,14,15)$. The bullae contain acantholytic cells with eosinophilic cytoplasm, pyknotic nuclei perinuclear halo, and serum. In older lesions, the dermis shows mild to moderate inflammatory infiltrate with lymphocytes, macrophages, along with eosinophils or neutrophils. The pattern of spongiosis in PV is eosinophilic or neutrophilic and appears in the very early stage of the disease, when acantholysis is absent (Table III) $(11,14,16,17)$. Subsequently, newly formed vacuoles and foci of acantholysis become confluent, resulting in fissures and bullae. However, eosinophilic and neutrophilic spongiosis, when present, is not specific to PV, the reaction being common to various other diseases $(11,14)$.

Pemphigus foliaceus (PF). In PF, IgG autoantibodies are targeting desmoglein1 (Dsg1). This subtype of pemphigus exhibits characteristic superficial and fragile bullae, which usually disappear in clinical examination, leaving an erosion post-examination. The mature PF lesion shows an intraepidermal bulla within spinous and granular layers. As in PV, the early stage of PF shows neutrophilic or eosinophilic spongiosis (Table III) $(11,16,17)$. Sometimes, PF can transform into PV, changing its clinicopathological and immunological profile.

The definite diagnosis requires, as in PV, supplementary immunofluorescence techniques as well as ELISA, besides histopathological examination $(10,11)$.

Paraneoplastic pemphigus (PNP). PNP presents IgG autoantibodies targeting cytosolic plakin proteins (desmoplakins1 and 2, periplakin, envoplakin), Dsg1, Dsg3, BP antigen1 (BP230), Dsc1-3, and $\alpha$-2-macroglobulin-like protein 1 (A2ML1) (10).

Within the spectrum of benign and malignant neoplasms, commonly lymphoproliferative malignancies, the histopathological features of PNP are associated with the lesion morphology, with an overlap of histological patterns. PNP consists of an interface dermatitis with few acanthotic areas and necrotic keratinocytes. This entity includes three histopathological patterns, as follows: i) intraepidermal suprabasal acantholytic bullae; ii) intraepidermal suprabasal acantholytic bullae with basal vacuolar degeneration; iii) nonspecific interface dermatitis with a thinned/hyperplastic epidermis, basal vacuolar degeneration, hypergranulosis, necrotic keratinocytes, and band-like lymphocytes (11). Eosinophilic spongiosis may be the initial histological feature in PNP, according to some studies $(14,18)$. However, because of its heterogeneity, the diagnosis requires confirmation by immunofluorescent and immunoblot studies (11).

IgA pemphigus. IgA pemphigus represents a rare entity of this group of bullous dermatoses (12). According to the site of bullae, there are two types of IgA pemphigus, namely 
Table III. Spongiosis patterns in autoimmune bullous dermatoses.

Spongiotic patterns

Autoimmune bullous dermatoses (ABD)

Eosinophilic

Neutrophilic

Eosinophilic and

spongiosis

spongiosis

neutrophilic spongiosis
Intraepidermal ABD

Pemphigus vulgaris (early stages)

Pemphigus foliaceus (early stages)

Paraneoplastic pemphigus

IgA pemphigus

Pemphigus herpetiformis

Subepidermal ABD

Bullous pemphigoid (early urticarial stage)

Gestational pemphigoid (early urticarial stage)

Cicatriceal pemphigoid (early urticarial stage)

Linear IgA disease (early urticarial stage)

Lichen planus pemphigoides

Dermatitis herpetiformis

Epidermolysis bullosa acquisita (early urticarial stage)

Anti-p200 pemphigoid

$\begin{array}{ccc}+ & + & +/- \\ + & + & - \\ +/- & - & - \\ - & + & - \\ + & + & + \\ + & & \\ + & - & - \\ -/+ & - & - \\ + & - & - \\ - & + & + \\ - & - & - \\ + & - & - \\ -/+ & + & -\end{array}$

- absent; + present; -/+ rarely present, +/- usually present. intraepidermal neutrophilic dermatosis (IEN) and subcorneal pustular dermatosis (SPD). IEN is characterized by IgA autoantigens against Dsg3 and Dsg1, while IgA autoantibodies target desmocollin1 (Dsc1) in SPD $(10,11)$.

Clinically, IgA pemphigus shows pustules, and histologically, both types identified show intraepidermal, subcorneal or spinous layer bullae, with hyperplastic epidermis, acantholytic keratinocytes, neutrophilic spongiosis, superficial inflammatory infiltrate with lymphocytes, neutrophils, and eosinophils, features which are also observed in other dermatoses (Table III) $(11,17,19)$. However, the most accurate diagnostic test remains direct immunofluorescence (10).

Pemphigus herpetiformis $(\mathrm{PH})$. $\mathrm{PH}$ is a rare variant with clinical features of herpetiform dermatitis, and histological and immunofluorescent features of pemphigus (11). PH shows IgG and C3 deposits, with anti-epithelial and anti-desmoglein1 (Dsg1) antibodies (20). The clinical aspects may vary from erythematous, pruriginous, vesicular or papular lesions, exhibiting herpetiform arrangement. The histopathological picture is nonspecific, showing eosinophilic spongiosis, the most characteristic spongiotic pattern for this dermatosis (11), as well as neutrophilic spongiosis, or a mixed eosinophilic-neutrophilic spongiosis, with acantholytic cells, intraepidermal vesicles, and papillary neutrophilic microabcesses (Table III) $(11,14,21)$. Early urticarial stages of $\mathrm{PH}$ may also present neutrophilic and eosinophilic spongiosis (16). The mature $\mathrm{PH}$ lesions show intraepidermal bullae, with various locations. The final diagnosis requires the corroboration of histopathological, clinical, and immunological techniques (11).

Subepidermal autoimmune bullous dermatoses. Subepidermal autoimmune bullous dermatoses, with autoantibodies against basement membrane components, represent a heterogeneous group, which include bullous pemphigoid (BP), gestational pemphigoid/herpes gestationis $(\mathrm{GP} / \mathrm{HG})$, mucous membrane or cicatricial pemphigoid (MMP/CP), linear IgA dermatosis, and lichen planus pemphigoides, as the pemphigoid group of bullous diseases. Other distinct autoimmune dermatoses are dermatitis herpetiformis (DH) and epidermolysis bullosa acquisita (EBA), as well as anti-p200 pemphigoid, a new entity, considered as part of the subepidermal autoimmune bullous dermatoses by some authors (10-12).

Bullous pemphigoid (BP). BP is the most common entity from this group, which affects especially elderly people, being characterized by circulating antibodies against hemidesmosomal proteins, as BP antigen1 (BPAG/BP230) and BPAG2 (BP180) $(10,11,22,23)$. Direct immunofluorescence represents the gold standard for diagnosis, revealing linear $\mathrm{C} 3, \mathrm{IgG}$, $\operatorname{IgA}, \operatorname{IgE}$ or $\operatorname{IgM}$, along the dermo-epidermal junction in perilesional biopsies (10). The histopathological examination reveals different morphological aspects, according to the stage of the lesion (24). In early or urticarial BP, the histological picture draws attention by eosinophilic spongiosis, with dermal edema, pseudovacuolar change, and perivascular inflammatory infiltrate dominated by lymphocytes, histiocytes, and numerous eosinophils (Table III) $(11,24)$. In this regard, one study reported a group of patients with clinical signs suggestive of BP, but with no characteristic histopathological and serological features. Biopsies showed in these cases only eosinophilic spongiosis, demonstrating either a particular form of bullous eczema or a BP with a prolonged incipient stage (25). BP shows subepidermal bullae with lymphocytes, eosinophils, neutrophils, plasma, and fibrin, in mature lesions (11). 
Gestational pemphigoid/Herpes gestationis (GP/HG). GP represents an autoimmune bullous dermatosis, which can occur during the second half of the pregnancy or in puerperium $(10,11,26,27)$. Recent studies have revealed the autoimmune pathogenesis, by identification of IgG autoantibodies against placental BP180 and, rarely, against BP230 $(10,11)$.

GP shares histological features with BP due to their similarities in the pathogenic mechanism, displaying various morphology according to the lesion age. Thus, there is no difference between these two entities, especially in early urticarial stages, which show eosinophilic spongiosis, subepidermal bullae, marked papillary edema, and dermal inflammatory infiltrate with lymphocytes, eosinophils, and occasional neutrophils (Table III). The bullae have the same content as in BP, with flat or necrotic overlying epidermis $(10,11)$. Direct immunofluorescence, ELISA and immunoblotting complement are sometimes necessary to certify the histopathologic diagnosis because of the high degree of heterogeneity and nonspecific morphological features (11).

Moreover, BP and PG could both represent intraepidermal and subepidermal autoimmune bullous dermatoses because extensive spongiosis can lead to the formation of epidermal bullae (11).

Mucous membrane pemphigoid/Cicatricial pemphigoid $(M M P / C P) . \mathrm{MMP}$ or $\mathrm{CP}$ represents a rare disease which stands out by autoantibodies against multiple basement membrane antigens, such as BP180, type VII collagen (COL7), $\beta 4$ integrin subunit, laminin5 (laminin332, epiligrin), and laminin6 (10).

The particularity of MMP is the main mucosal localization of bullae, with the skin being involved only in $25 \%$ of cases (9). The most affected areas are represented by the oral mucosa, closely followed by ocular lesions $(10,11,24)$.

Because of the numerous CP clinicopathological variants, it is more likely a general term comprising several diseases with similar clinical features and cicatrices, with oral and ocular mucous membrane tropism and with subepidermal bullae formation $(10,11)$.

Given the histological similarities with BP, CP represents a variant of bullous pemphigoides, although some authors report clinical, morphological, and immunological differences between these two entities $(11,28)$. Subepithelial blisters dominate the histological picture, with dermal inflammatory infiltrates containing lymphocytes, numerous eosinophils, and neutrophils, sometimes associated with fibrosis. Eosinophilic spongiosis occurs less frequently in CP (14). The histological and immunological heterogeneity make the $\mathrm{CP}$ diagnosis complex, always taking into account additional immunological testing $(10,11)$.

Linear IgA disease ( $L A D)$. LAD has a tendency to affect children, although an adult type linear $\operatorname{IgA}$ dermatosis is also described, which differs by the clinical presentation $(10,11)$. According to the immunofluorescent patterns and protein targets, there are two types of linear IgA disease: i) lamina lucida type, with two ectodomains of BP180, LABD97, and LAD-1 as target antigens and ii) sublamina densa type, with COL7, as well as other target antigens (10).

As in other autoimmune subepidermal bullous dermatoses, LAD shows eosinophilic, neutrophilic, or a combined spongiotic reaction pattern, with perivascular inflammatory infiltrate with lymphocytes, neutrophils, and eosinophils, as well as vacuolar degeneration of basement membrane zone, in early urticarial lesions (Table III) $(11,29)$. Although displaying typical formation of subepidermal bulla and a mixed dermal inflammatory infiltrate with neutrophils and eosinophils, the general histopathological picture is almost identical with that seen in dermatitis herpetiformis (DH), with a tendency to form papillary microabcesses (11).

Lichen planus pemphigoides (LPP). LPP represents a heterogeneous disease characterized by basal membrane autoantibodies directed against numerous antigens and the formation of bullous lesions, probably developed as a result of basal keratinocyte hydropic degeneration, in lichen planus (11). The histopathological features highlight subepidermal bullae associated with perivascular inflammatory infiltrate with lymphocytes and eosinophils, sometimes with characteristic morphological features of lichen planus, such as acanthotic epidermis with hypergranulosis and a band-like lymphocytic infiltrate. Immunofluorescence, other immunological techniques, and clinical correlations are mandatory for LPP diagnosis (11).

Dermatitis herpetiformis (DH). DH is an IgA-mediated bullous dermatosis, which forms autoantibodies against epidermal transglutaminase (eTG) and against tissue transglutaminase (tTG) (10). The histopathological picture demonstrates subepidermal clefting and neutrophils in the dermal papillae, in most cases. The typical evolution of the lesions comprises an early perivascular and interstitial inflammatory infiltrate with lymphocytes, neutrophils, sometimes eosinophils, and papillary dermis edema, followed by fibrin in papillary tips, and even abscesses, in cases with numerous neutrophils. In time, the subepidermal clefts transform into bullae, which are characteristic for mature lesions, while dermal infiltrate contains a high amount of eosinophils (11).

Because of nonspecific DH histopathology and common morphological changes with other bullous dermatoses, the diagnosis confirmation should comprise direct immunofluorescence and electron microscopy, which highlight the granular IgA pattern, in dermal papillae $(11,30,31)$.

Epidermolysis bullosa acquisita (EBA). EBA represents a rare and chronic bullous dermatosis, characterized by autoantibodies against COL7, with subepidermal blister formation $(10,32)$. EBA can exhibit different clinical forms, as well as variable and nonspecific histopathological features (11). Eosinophilic or neutrophilic spongiosis, associated with perivascular inflammatory infiltrate with lymphocytes, neutrophils, and eosinophils characterizes only the early urticarial lesions, the findings being similar with those seen in other bullous dermatoses (28) (Table III). The variable clinical presentations correspond to different histological patterns. Thus, in classic type, the morphological features are bullae, associated with low perivascular and interstitial lymphocytic infiltrate. Inflammatory variant shows an abundant inflammatory infiltrate, with lymphocytes, eosinophils, and neutrophils, this form being similar with BP or with the mucous form of EBA. Other variants stand out by papillary neutrophilic 
abscesses, while non-inflammatory type lacks inflammation at the trauma sites $(10,11)$. Recurrent forms display fibrosis and milia (33). The diagnosis confirmation requires additional immunological testing and confirmation by electron and immunoelectron microscopy $(11,34)$.

Anti-p200 pemphigoid. Anti-p200 pemphigoid represents a distinct subepidermal autoimmune bullous dermatosis, which shares clinical features with linear IgA bullous dermatosis, BP, or DH (11). Recent studies have revealed autoantibodies against recombinant laminin $\gamma 1$ in anti-p200 pemphigoid $(11,35)$.

The histopathological picture does not have specific features, as subepidermal bullae, along with inflammatory infiltrate with occasional papillary microabscesses are also seen in dermatitis herpetiformis or linear IgA disease $(11,36)$. Few studies are also reporting an additional eosinophilic spongiosis $(11,37,38)$.

\section{Conclusions}

Although spongiosis exhibits different reaction patterns, only eosinophilic spongiosis and neutrophilic spongiosis are completing the histological picture of the autoimmune bullous dermatoses. While almost all the intraepidermal bullous diseases display eosinophilic and neutrophilic, or a mixed pattern of spongiosis, in early stages, only half of the subepidermal bullous dermatoses show these patterns of spongiosis in early urticarial lesions.

The occurrence of spongiosis limited to the early stages of these diseases emphasizes the transient nature of this process, a feature that can mirror the specific pathogenesis of these autoimmune entities.

Considering these findings, as well as the heterogeneity and non-specificity of the histopathological features of these diseases, the diagnosis is very complex, requiring clinicopathological correlations, as well as additional techniques, such as electron microscopy, immunofluorescence, immunohistochemistry or molecular biology. All of these diagnostic methods may contribute to a thorough understanding of the pathogenic mechanisms, as a fundamental source of possible classification refinement of these autoimmune bullous dermatoses.

\section{Acknowledgements}

Not applicable.

\section{Funding}

No funding was received.

\section{Availability of data and materials}

The data used to support the findings of this study are included in the article and are also available from the corresponding author upon request.

\section{Authors' contributions}

$\mathrm{RAB}$ and $\mathrm{CA}$ conceived and designed the review. RAB reviewed the literature data and wrote the manuscript. CA contributed to the drafting of the manuscript and revised it critically for important intellectual content in light of the literature data. IDC revised it critically for important intellectual content in light of the literature data. LL, AG, SEG, and TAB were responsible for collecting and analyzing the data. All authors read and approved the final version of the manuscript for publication.

\section{Ethics approval and consent to participate}

Not applicable.

\section{Patient consent for publication}

Not applicable.

\section{Competing interests}

The authors declare that they have no competing interests.

\section{References}

1. Murphy M and Grant-Kels JM: Spongiotic Dermatitis. In: The Textbook Dermatopathology. 3rd edition. Barnhill R (ed). Medical Publishing Division, McGraw-Hill Professional, New York, NY, pp15-18, 2010.

2. Weedon D: An approach to the interpretation of skin biopsies. In: Weedon's Skin Pathology. Livingstone C (ed). Elsevier, London, pp6-7, 2010.

3. Weedon D: The spongiotic reaction pattern. In: Weedon's Skin Pathology. Livingstone C (ed). Elsevier, London, pp93-97, 2010.

4. Trautmann A, Altznauer F, Akdis M, Simon HU, Disch R, Bröcker EB, Blaser K and Akdis CA: The differential fate of cadherins during T-cell-induced keratinocyte apoptosis leads to spongiosis in eczematous dermatitis. J Invest Dermatol 117: 927-934, 2001.

5. Holden CA and Berth-Jones J: Eczema, lichenification, prurigo and erythroderma. In: Rook's Textbook of Dermatology. Blackwell Science Ltd., Oxford, pp11-15, 2004.

6. Ohtani T, Memezawa A, Okuyama R, Sayo T, Sugiyama Y, Inoue $S$ and Aiba $S$ : Increased hyaluronan production and decreased E-cadherin expression by cytokine-stimulated keratinocytes lead to spongiosis formation. J Invest Dermatol 129: 1412-1420, 2009.

7. Wolff K, Kibbi AG and Mihm MC: Basic pathologic reactions of the skin. In: Dermatology in General Medicine. McGraw-Hill, New York, pp30-43, 2003.

8. Neis MM, Peters B, Dreuw A, Wenzel J, Bieber T, Mauch C, Krieg T, Stanzel S, Heinrich PC, Merk HF, et al: Enhanced expression levels of IL-31 correlate with IL-4 and IL-13 in atopic and allergic contact dermatitis. J Allergy Clin Immunol 118: 930-937, 2006.

9. Zeidi M, Chen KL, Patel B, Ravishankar A, Lim R and Werth VP: Increased MxA protein expression and dendritic cells in spongiotic dermatitis differentiates dermatomyositis from eczema in a single-center case-control study. J Cutan Pathol 48: 364-373, 2021 .

10. Harrell J, Rubio XB, Nielson C, Hsu S and Motaparthi K: Advances in the diagnosis of autoimmune bullous dermatoses. Clin Dermatol 37: 692-712, 2019.

11. Radoš J: Autoimmune blistering diseases: Histologic meaning. Clin Dermatol 29: 377-388, 2011.

12. Baum S, Sakka N, Artsi O, Trau H and Barzilai A: Diagnosis and classification of autoimmune blistering diseases. Autoimmun Rev 13: 482-489, 2014.

13. Schmidt E, Dähnrich C, Rosemann A, Probst C, Komorowski L, Saschenbrecker S, Schlumberger W, Stöcker W, Hashimoto T, Bröcker EB, et al: Novel ELISA systems for antibodies to desmoglein 1 and 3: Correlation of disease activity with serum autoantibody levels in individual pemphigus patients. Exp Dermatol 19: 458-463, 2010. 
14. Morais KL, Miyamoto D, Maruta CW and Aoki V: Diagnostic approach of eosinophilic spongiosis. An Bras Dermatol 94: 724-728, 2019

15. Laws PM, Heelan K, Al-Mohammedi F, Walsh S and Shear NH: Pemphigus herpetiformis: A case series and review of the literature. Int J Dermatol 54: 1014-1022, 2015.

16. Megahed M: Intraepidermal blistering diseases. In: Histopathology of Blistering Diseases With Clinical, Electron Microscopic, Immunological and Molecular Biological Correlations. Megahed M (ed). Springer-Verlag, Heidelberg, pp49-153, 2004.

17. Hong W, Brandling-Bennett HA and Harrist TJ: Noninfectious vesiculobullous and vesiculopustular disease. In: Lever's histopathology of the skin. 10th edition. Elder D, Elenitsas R, Jaworosky C and Johnson B (eds). Lippincott-Raven, Philadelphia, pp246-267, 2010.

18. Gallo E, García-Martín P, Fraga J, Teye K, Koga H, Hashimoto T and Garcia-Diez A: Paraneoplastic pemphigus with eosinophilic spongiosis and autoantibodies against desmocollins 2 and 3. Clin Exp Dermatol 39: 323-326, 2014.

19. Kopp T, Sitaru C, Pieczkowski F, Schneeberger A, Födinger D, Zillikens D, Stingl G and Karlhofer FM: IgA pemphigus-occurrence of anti-desmocollin 1 and anti-desmoglein 1 antibody reactivity in an individual patient. J Dtsch Dermatol Ges 4: 1045-1050, 2006 (In English, German).

20. Fuentes-Finkelstein P, Barnadas M, Gelpi C and Puig L: Pemphigus herpetiformis with progression to pemphigus foliaceus: A case report. Actas Dermosifiliogr 105: 526-528, 2014 (In English, Spanish).

21. Duarte IB, Bastazini I Jr, Barreto JA, Carvalho CV and Nunes AJ: Pemphigus herpetiformis in childhood. Pediatr Dermatol 27: 488-491, 2010.

22. Taghipour K and Perera GK: Autoimmune blistering skin diseases. Medicine 45: P405-P412, 2017.

23. Langan SM, Smeeth L, Hubbard R, Fleming KM, Smith CJ and West J: Bullous pemphigoid and pemphigus vulgaris-incidence and mortality in the UK: population based cohort study. BMJ 337: a180, 2008.

24. Rapini RP: Subepidermal vesicular diseases In: Practical Dermatopathology. Elsevier Saunders, Philadelphia, pp93-99, 2012.

25. Atteh G, Cole EF, Perricone AJ and Feldman RJ: Bullous eczema presenting as bullous pemphigoid-like eruption: A case series. JAAD Case Rep 10: 34-37, 2021.

26. Patsatsi A, Marinovic B and Murrell D: Autoimmune bullous diseases during pregnancy: Solving common and uncommon issues. Int J Womens Dermatol 5: 166-170, 2019.

27. Moore EM and Werth VP: Pemphigoid gestationis. In: Autoimmune Bullous Diseases: Approach and Management. Sami N (ed). Springer, New York, NY, pp149-162, 2016.
28. Megahed M: Subepidermal blistering diseases. In: Histopathology of Blistering Diseases With Clinical, Electron Microscopic, Immunological and Molecular Biological Correlations. Springer-Verlag, Berlin Heidelberg, pp255-275, 2004.

29. Rao CL and Hall RP: Linear immunoglobulin a dermatosis and chronic bullous disease of childhood. In: Fitzpatrick's dermatology in general medicine. Wolff K, Goldsmith LA, Katz SI, Gilchrest BA, Paller AS and Leffell DJ (eds). McGraw Hill, New York, NY, pp485-490, 2008.

30. Antiga E, Maglie R, Quintarelli R, Verdelli A, Bonciani D, Bonciolini V and Caproni M: Dermatitis herpetiformis: Novel perspectives. Front Immunol 10: 1290, 2019.

31. Clarindo MV, Possebon AT, Soligo EM, Uyeda H, Ruaro RT and Empinotti JC: Dermatitis herpetiformis: Pathophysiology, clinical presentation, diagnosis and treatment. An Bras Dermatol 89: 865-875, 2014.

32. Ishii N, Hamada T, Dainichi T, Karashima T, Nakama T, Yasumoto S, Zilikens D and Hashimoto T: Epidermolysis bullosa acquisita: What's new? J Dermatol 37: 220-230, 2010.

33. Vorobyev A, Ludwig RJ and Schmidt E: Clinical features and diagnosis of epidermolysis bullosa acquisita. Expert Rev Clin Immunol 13: 157-169, 2017.

34. Chen M, Hallel-Halevy D, Nadelman C and Woodley DT: Epidermolysis bullosa acquisita. In: Autoimmune Disease of the Skin. Pathogenesis, diagnosis, management. Springer, New York, NY, pp109-132, 2005.

35. Kridin K and Ahmed AR: Anti-p200 pemphigoid: A systematic review. Front Immunol 10: 2466, 2019.

36. Rose C, Weyers W, Denisjuk N, Hillen U, Zillikens D and Shimanovich I: Histopathology of anti-p200 pemphigoid. Am J Dermatopathol 29: 119-124, 2007.

37. Meijer JM, Diercks GF, Schmidt E, Pas HH and Jonkman MF: Laboratory diagnosis and clinical profile of anti-p200 pemphigoid. JAMA Dermatol 152: 897-904, 2016.

38. García-Díez I, Martínez-Escala ME, Ishii N, Hashimoto T, Mascaró Galy JM, Pujol RM and Herrero-González JE: Usefulness of a simple immunohistochemical staining technique to differentiate anti-p200 pemphigoid from other autoimmune blistering diseases: A report of 2 cases. Actas Dermosifiliogr 108: e1-e5, 2017 (In English, Spanish). 